\title{
Perforated diverticulitis in a patient with very proximal jejunal diverticula
}

\author{
Constantinos Avgoustou ${ }^{1 *}$, Dimitrios Velecheris ${ }^{1}$
}

'Department of Surgery, General Hospital of Nea Ionia “Konstantopouleio-Agia Olga”, Athens, Greece.

\section{To Cite}

Avgoustou C, VelecherisnD. Perforated diverticulitis in a patient with very proximal jejunal diverticula. J Gastric Surg 2020; 2(3): 102-105

\section{Publication history}

Received: July 30, 2020

Accepted: August 4, 2020

Article in press: August 30, 2020

Published online: August 31, 2020

\section{*Correspondence to}

Dr. Constantinos Avgoustou

Department of Surgery

General Hospital of Nea Ionia

"Konstantopouleio-Agia Olga"

Agias Olgas 3, 14233 Nea Ionia

Athens, Greece

avgoustouk@yahoo.gr

\section{ABSTRACT \\ Background:}

We report a case of an elderly with peritonitis due to perforated jejunal diverticulitis, and we highlight the diagnostic evaluation and treatment alternatives.

\section{Case presentation:}

A 92-year-old woman was transferred to the Emergency Dept. with abdominal pain and vomiting for the past 12 hours. Physical examination revealed diffuse pain, abdominal distension, rebound tenderness and bowel silence. She was febrile, tachycardic, tachypneic, hypotensive and anuric. Blood gas estimation showed metabolic acidosis. She fulfilled the criteria of septic shock. At presentation, she was mildly malnourished. From her medical history, she had cardiac arrythmias, hypertension and diabetes mellitus under proper medication, and laparoscopic cholecystectomy. Laboratory investigations revealed Hct 44.6\%, WBC 12.500/dL, glucose $300 \mathrm{mg} / \mathrm{dL}$, creatinine $2.8 \mathrm{mg} / \mathrm{dL}$, CRP $405 \mathrm{mg} / \mathrm{L}$, and electrolyte deficit. Abdominal X-ray showed gastric, small intestinal and colonic gas, with no pneumoperitoneum or air-fluid levels. Chest/abdomen CT showed thickening of proximal jejunal loop and adjacent mesentery, and an extraluminal air bubble, suggesting possible perforation. The patient was given intense resuscitation and broad-spectrum antibiotics and underwent emergency laparotomy.

Results:

Four jejunal diverticula, sized 1-3 cm, were confined to a segment $12 \mathrm{~cm}$ long, located 6 $\mathrm{cm}$ from the Treitz ligament; the proximal diverticula was inflamed and perforated. The adjacent mesentery was inflamed and thickened; the bowel lumen remained open. We performed one-layer full-thickness suturing of the perforated diverticulum and omental patch closure. The patient was transferred intubated to ICU. E. Coli was isolated from peritoneal fluid cultures and antibiotic therapy was adjusted to antibiogram. The patient had a first bowel movement at day 5 and was extubated at day 21. She needed mild cardiopulmonary support and was discharged at day 30 .

\section{Conclusions:}

Jejunal diverticulitis is a challenging disorder since its rarity makes diagnosis difficult and, thus delayed. The perforation of jejunal diverticulitis requires emergent surgery and poses technical dilemmas.

Key words:

jejunal diverticulitis, perforation, emergency surgery. 


\section{Background}

Diverticulosis jejuni (DJ) is a very rare condition, and complicated diverticulosis jejuni (CDJ) is even rarer [18]. The clinical incidence of small intestine diverticula ranges from $0.06 \%$ to $1.3 \%[5,9,10]$, but results of contrast studies and autopsies showed an incidence of $0.5 \%-4.6 \%$ $[3,5,9-12]$. DJ is most frequently seen in the elderly, with a slight male preponderance $[5,9,11]$. Intestinal diverticula are pseudodiverticula of the pulsion type, they are usually multiple and mostly located in the proximal jejunum $[4,5,9,13]$. DJ is usually asymptomatic or may cause unspecific long-standing symptoms with a variable periodicity and severity $[1,3,5,6,9,10]$. Related complications, such as diverticulitis, hemorrhage, perforation and obstruction appear in about $10-30 \%$ of the patients, and increase morbidity and mortality [1,39,12,14-17]. Herein, we report a rare case of a 92-year old woman who had severe comorbidities, presented with peritonitis and in septic shock due to a jejunal diverticulum adjacent to the ligament of Treitz, inflamed and perforated in the mesentery, but was lucky to have a prompt diagnosis and a successful surgical treatment, along with a brief review of the literature.

\section{Case presentation}

A 92-year-old woman was transferred to the Emergency Dept. in severe condition, with onset of abdominal pain and vomiting 12 hours before. Physical examination revealed severe diffuse pain, abdominal distension, rebound tenderness and absence of bowel sounds. She was febrile $(37.90 \mathrm{C})$, tachycardic (110 beats/min), tachypneic (24 breaths/min), hypotensive (80/50 $\mathrm{mmHg}$ ) and anuric. Her blood gas estimation showed metabolic acidosis, with $\mathrm{pH}$ at 7.22, pO2 $55 \mathrm{mmHg}$, pCO2 $34 \mathrm{mmHg}$, [HCO3-] $18 \mathrm{mEq} / \mathrm{L}$ and Lac 20 $\mathrm{mEq} / \mathrm{L}$. She fulfilled the criteria of septic shock. The thin patient had a significant medical history with cardiac fibrillation, hypertension and diabetes mellitus; she was under suitable medication that included antithrombotics and antidiabetics per os. She was diagnosed as suffering from "altered bowel habits-gaseous dyspepsia" for the last 5 years. She was mostly bed-ridden for the last year, and mildly malnourished at presentation (albumin 2.8 gr/dL). No known history of sigmoid diverticulosis or other systemic disease was reported; she only had a history of laparoscopic cholecystectomy. Laboratory investigations revealed Hct 44.6\%, WBC 12.500/dL, glucose $300 \mathrm{mg} / \mathrm{dL}$, creatinine $2.8 \mathrm{mg} / \mathrm{dL}, \mathrm{CRP} 405$ $\mathrm{mg} / \mathrm{L}$, and mild electrolyte deficit. Abdominal X-ray showed gastric, small intestinal and colonic gas, with no pneumoperitoneum or air-fluid levels (Fig. 1). Immediate chest/abdomen CT showed thickening of a segment of the proximal jejunal loop, thickening and infiltration of the mesenteric fat adjacent to the descending colon, and the presence of an extraluminal air bubble isolated in the mesentery, suggesting a possible perforation (Fig. 2). The patient was given intense resuscitation with parenteral fluids and inotropic agents, as well as broad-spectrum antibiotics (piperacillin/tazobactam) in bolus doses and underwent emergent exploratory open laparotomy.

Four jejunal diverticula were confined to a segment $12 \mathrm{~cm}$ long, located $6 \mathrm{~cm}$ from the Treitz ligament; three were situated at the mesenteric border, the most proximal of which was inflamed and perforated, and the other one at the antimesenteric border, sized 1-3 cm (Fig. 3). The adjacent mesentery was severely inflamed and thickened, but the bowel lumen remained open. We performed one-layer full-thickness suturing at the base of the perforated diverticulum, and omental patch closure. The abdomen was irrigated, drained and closed. The patient was transferred intubated to ICU. E. Coli was isolated from peritoneal fluid cultures, and, at day 10, Pseudomonas aeruginosa from urine and bronchial cultures; antibiotics were suitably adjusted (ciprofloxacin, teicoplanin). The patient had a first bowel movement at day 5 and she was extubated at day 21 . She needed mild cardiopulmonary support and was discharged at day 30 . The patient's course is good during a follow-up of 12 months.

Informed consent was obtained from the patient.

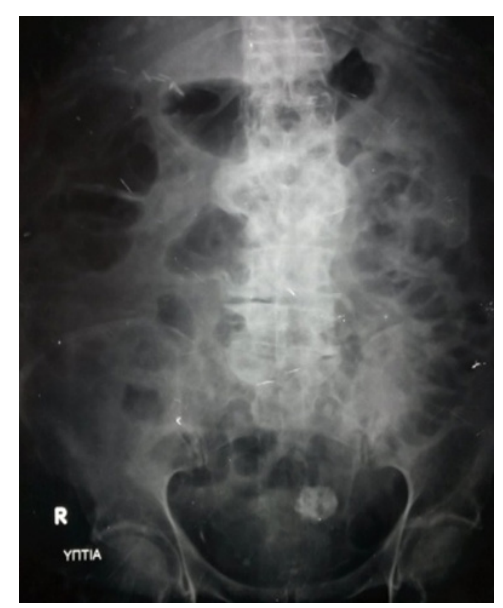

Figure 1: Abdominal x-ray of a 92-year-old woman with peritonitis, showing gastric, small intestinal and colonic gas. Airfluid levels are not obvious due to supine position of patient.

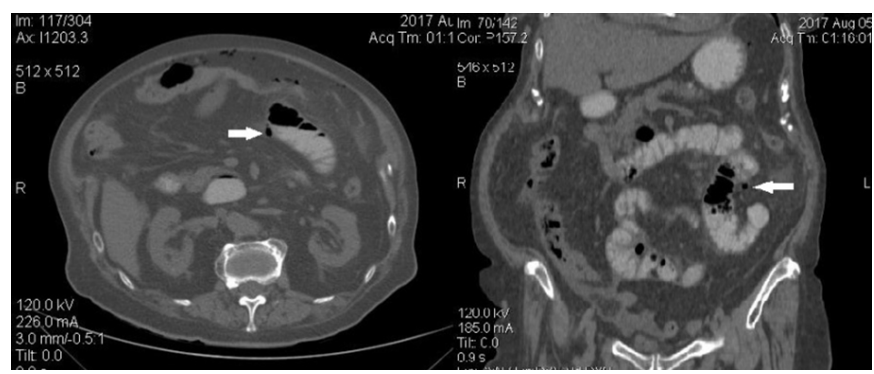

Figure 2: Chest-abdomen CT of a 92-year-old woman with peritonitis, showing thickening of a segment of the proximal jejunal loop and the adjacent mesenteric fat and presence of an extraluminal air bubble in the mesentery (white arrows).

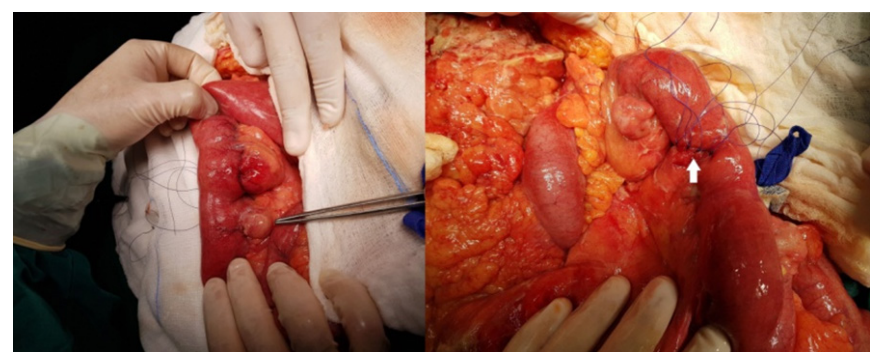

Figure 3: Intraoperative findings: Forceps point at mesenteric jejunal diverticula (posterior side), white arrow points at the one-layer fullthickness suturing of the perforated diverticulum. Adjacent inflamed mesentery is evident. 


\section{Discussion}

Small intestine diverticula other than Meckel are acquired and false outpouchings, characterized by herniation of the mucosa and submucosa through the muscle coat in places of minor resistance, typically the mesenteric site, where blood vessels penetrate the intestinal wall [2,9$12,18]$. Possible etiology for their formation is suggested to be the intestinal dyskinesis, probably due to motor dysfunction of the smooth muscle or the myenteric plexus, either associated with a systemic disease (i.e. sclerosis, amyloidosis) or not, that leads to high segmental intraluminal pressure $[5,9,10,12,18]$.

DJ is usually an asymptomatic disorder or causes chronic unspecific symptoms, like intermittent postprandial abdominal pain, nausea, vomiting, borborygmi, alternating diarrhea and constipation, anemia, malabsorption and weight loss

[1-

$3,5,6,9,10,12,18$ ]. A triad has been described by Edwards in such patients as "flatulent dyspepsia: epigastric pain, abdominal discomfort, flatulence one or two hours after meals" $[10,12]$. In about $10-30 \%$ of the patients, acute lifethreatening complications, like diverticulitis, intestinal hemorrhage, perforation, intraabdominal abscess and obstruction (due to volvulus, intussusception, adhesions or enterolith) may occur [1-3,5,7-10,14-20]. Even though the jejunal and the ileal diverticula are seen 3 times less frequently than those found in the duodenum, they lead to complications 4 times more often [10]. In case of CDJ, the patients are seriously ill and this should be taken under consideration when deciding the proper emergent management, especially since this condition affects more frequently the elderly $[1,3,5,7,9,12,16,18,20]$. As a result of its rarity and diffuse symptomatology, often masking other disorders, CDJ is a challenging acute condition from the diagnostic perspective $[1,3,6,18]$. Still today, diagnosis of CDJ is most frequently confirmed intraoperatively, sometimes after considerable delay that increases morbidity and mortality $[3,5,9,11,18]$. Delayed diagnosis of perforation can be fatal in up to $40 \%$ of patients [9]. However, high index of suspicion and proper imaging may help timely diagnosis [8]. Plain films may show air-fluid levels, dilated intestinal loops or free air $[2,9,10,18]$. Ultrasound may indicate thickening of the walls of intestinal loops, increased echogenicity in the surrounding fat tissue and free fluids [2,9]. CT imaging is the most reliable tool and may show ovoid diverticula containing contrast-air-fluids, thickening of the walls of jejunum and the diverticulum, increased density of the surrounding mesentery and, in case of perforation, free air and fluid or a loculated collection $[2,3,7-10,16,17,20,21]$.

CDJ presenting with acute abdomen generally requires surgical treatment, whereas in cases with local mild inflammation and in the absence of other complications such as hemorrhage, obstruction and perforation or abscess, conservative management (i.e. bowel rest, antibiotics) can be attempted [1,2,4,7-10,12,13,16,19,21]. Surgical resection that should be limited to the involved jejunal section, based on the principles of septic surgery implying immediate decontamination and healthy wound edges, and primary anastomosis is the treatment of choice $[1,3,4,7,9,14,20]$. Other surgical techniques, such as invagination of the diverticulum with a suture, or suturing the perforation with omental patch closure, are generally avoided and rarely used in cases with no hemorrhage or obstruction [9]. Taking into account, firstly, the fact that excision and anastomosis on this location of jejunal diverticulitis with severely inflamed and thickened accompanying mesentery could be harmful, secondly, the finding that the bowel lumen was not obstructed, and, thirdly, the need for a timesaving operation, we opted for suturing the perforation with omental patch closure for our frail elder patient, providing that suturing did not occlude the bowel lumen.

\section{Conclusion}

In elderly patients with peritonitis due to a perforated diverticulum, diverticular disease, causing increased morbidity and mortality, does not always originate from the colon, but may also originate from the jejunum. CDJ may lead to diagnostic and therapeutic delay, as well as it may pose technical dilemmas related to its location. Prompt diagnosis of CDJ is essential and emergent surgery is mandatory.

\section{Acknowledgements \\ None}

\section{Contributors}

CA, DV conceptualized and designed the study, acquired, and analyzed data, interpreted the study results, drafted the manuscript, and critically revised the final version of the manuscript.

\section{Funding}

No funding was received for this study.

\section{Competing interests}

No benefits in any form have been received or will be received from a commercial party related directly or indirectly to the subject of this article.

\section{Availability of data and materials}

Further information is available from the corresponding author on reasonable request.

\section{Ethics approval}

Written informed consent was obtained from the patient or the relatives for publication of this case report and accompanying images.

\section{Provenance and peer review}

Not commissioned; externally peer reviewed.

\section{Open access}

This is an Open Access article distributed in accordance with the Creative Commons Attribution NonCommercial (CC BY-NC 4.0) license, which permits others to distribute, remix, adapt, build upon this work noncommercially, and license their derivative works on different terms, provided

the original work is properly cited and the use is non-commercial. See: http://creativecommons.org/ licenses/by-nc/4.0/ 


\section{References}

[1] Johnson KN, Fankhauser GT, Chapital AB, Merritt MV, Johnson DJ. Emergency management of complicated jejunal diverticulosis. Am Surg. 2014;80:600-3.

[2] Fidan N, Mermi EU, Acay MB, Murat M, Zobaci E. Jejunal diverticulosis presented with acute abdomen and diverticulitis complication: A case report. Pol J Radiol. 2015;80:532-5.

[3] Téoule P, Birgin E, Zaltenbach B, et al. A retrospective, unicentric evaluation of complicated diverticulosis jejuni: Symptoms, treatment, and postoperative course. Front Surg. 2015;2:57.

[4] Khan HS, Ayyaz M. Jejunal diverticulosis presenting as an acute emergency. J Coll Physicians Surg Pak. 2015;25 Suppl 1:S20-1.

[5] Mohi RS Ms, Moudgil A Ms, Bhatia SK Ms, Seth K Ms, Kaur T Ms. Complicated jejunal diverticulosis: Small bowel volvulus with obstruction. Iran J Med Sci. 2016;41:548-51.

[6] Walter BM, Winker J, Wagner M, Jung A, Strebel H, Born $\mathrm{P}$. Complicated jejunal diverticulosis - a rare but important diagnosis to consider in abdominal pain: a report of three cases. Z Gastroenterol. 2016;54:562-5.

[7] Harbi H, Kardoun N, Fendri S, et al. Jejunal diverticulitis. Review and treatment algorithm. Presse Med. 2017;46(12 Pt 1):1139-43.

[8] Lebert P, Millet I, Ernst $O$, et al.Acute jejunoileal diverticulitis: Multicenter descriptive study of 33 patients. AJR Am J Roentgerol. 2018;210:1245-51.

[9] Kassir R, Boueil-Bourlier A, Baccot S, et al.Jejuno-ileal diverticulitis: Etiopathogenicity, diagnosis and management. Int J Surg Case Rep. 2015;10:151-3

[10] Aydın E, Yerli H, Avcı T, Yılmaz T, Gülay H. One of the rare causes of acute abdomen leading to subileus: Jejunal diverticulitis. Balkan Med J. 2016;33:354-6.

[11] Patel VA, Jefferis H, Spiegelberg B, Iqbal Q, Prabhudesai A, Harris S. Jejunal diverticulosis is not always a silent spectator: a report of 4 cases and review of the literature. World J Gastroenterol. 2008;14:5916-9.

[12] Falidas E, Vlachos K, Mathioulakis S, Archontovasilis F, Villias C. Multiple giant diverticula of the jejunum causing intestinal obstruction: report of a case and review of the literature. World J Emerg Surg. 2011;6:8.

[13] Horesh N, Klang E, Gravetz A, et al. Jejunal diverticulitis. J Laparoendosc Adv Surg Tech A. 2016; 26:596-9.

[14] Longo WE, Vernava AM 3rd. Clinical implications of jejunoileal diverticular disease. Dis Colon Rectum 1992;35:381-8.

[15] Chow DC, Babaian M, Taubin HL. Jejunoileal diverticula. Gastroenterologist 1997 ;5:78-84. PMID:9074921Wilcox RD, Shatney CH.Am Surg. 1990;56:222-5.

[16] Veen M, Hornstra BJ, Clemens CH, Stigter H, Vree R. Small bowel diverticulitis as a cause of acute abdomen. Eur J Gastroenterol Hepatol. 2009;21:123-5.

[17] França M, Certo M, Silva D, Peixoto C, Varzim P. Elderly patient with acute, left lower abdominal pain: perforated jejunal diverticulitis. Eur Radiol. 2010;20:2541-5.

[18] Natarajan K, Phansalkar M, Varghese RG, Thangiah G. Jejunal diverticulosis with perforation - a challenging differential diagnosis of acute abdomen: case report. J Clin Diagn Res. 2015;9:ED034.

[19] Levack MM, Madariaga ML, Kaafarani HM. Non-operative successful management of a perforated small bowel diverticulum. World J Gastroenterol. 2014;20:18477-9.

[20] López Marcano AJ, Ramia JM, De la Plaza Llamas R, Alonso S, Gonzales Aguilar JD, Kühnhardt Barrantes AW. [Complicated jejunoileal diverticular disease: a 12 cases' serie and literature review]. Rev Gastroenterol Peru. 2017;37:240-245.

[21] Macari M, Faust M, Liang H, Pachter HL. CT of jejunal diverticulitis: imaging findings, differential diagnosis, and clinical management. Clin Radiol. 2007;62:73-7. 